\title{
Repair of a Chronic Triceps Tendon Rupture in a Dog Using an Autogenous Thoracolumbar Fascia Onlay Graft
}

\author{
Christopher J. Wood ${ }^{10}$ Ricky G. Cashmore ${ }^{2}$ \\ 1 VCA Canada Alta Vista Animal Hospital, Ottawa, Ontario, Canada \\ ${ }^{2}$ Veterinary Specialist Surgery Australia, Sandringham, Victoria, \\ Australia
}

\begin{abstract}
Address for correspondence Christopher J. Wood, DVM, VCA Canada Alta Vista Animal Hospital, 2616 Bank Street, Ottawa K1T 1M9, Ontario, Canada (e-mail: woodchrisjames@gmail.com).
\end{abstract}

VCOT Open 2021;4:e32-e36.

\begin{abstract}
Keywords

- tendon repair

- tendon injury

- lameness

- dogs

A 5-year-old $38 \mathrm{~kg}$ entire male German Shepherd dog was referred for persistent nonweight bearing left thoracic limb lameness 5 weeks following failed triceps tendon repair. Physical exam revealed complete incompetence of the triceps mechanism with a large palpable defect proximal to the olecranon and when the shoulder was fixed in extension, the elbow could be fully flexed. A purulent draining tract was present on the caudolateral aspect of the distal brachium. Staged tendon repair was delayed until resolution of infection to allow for improved healing. Culture following surgical exploration of the traumatized area with resection of sinus tract and interposed fibro-granulomatous tissue yielded no growth. Definitive triceps brachii tendon repair was augmented with an autogenous thoracolumbar fascia onlay graft. Postoperatively, the repair was protected with a spica splint and the left thoracic limb immobilized with the elbow maintained in an extended position for 6 weeks. Re-evaluation 7 months later revealed the patient to be free of lameness. Disruption of the triceps brachii tendon is a rarely reported tendon injury with chronic disruption of triceps tendon associated with a guarded prognosis. To the authors knowledge, successful surgical repair of tendinous injury incorporating the use of an autogenous thoracolumbar fascia onlay graft has not previously been described.
\end{abstract}

\section{Introduction}

Disruption to the triceps brachii tendon is a rarely reported tendon injury in companion animals, with most triceps tendon disruptions secondary to avulsion. ${ }^{1}$ Treatment outcomes have been limited to only a few descriptive studies in dogs. ${ }^{2-10}$ This report describes successful treatment of a chronic complete laceration of the triceps brachii tendon in a $38 \mathrm{~kg}$ entire male German Shepherd. Surgical management included staged debridement, with delayed primary repair supported by an autogenous thoracolumbar fascia (TLF) onlay graft. To the best of the authors' knowledge, successful surgical repair of tendinous injury incorporating the use of a TLF graft has not previously been described.

received

October 21, 2020 accepted after revision January 16, 2021
DOI https://doi.org/ $10.1055 / \mathrm{s}-0041-1725056$ ISSN 2625-2325.

\section{Case History}

A 5-year-old $38 \mathrm{~kg}$ entire male German Shepherd dog was referred for persistent non-weight bearing left thoracic limb lameness 5 weeks after failed triceps tendon repair. The inciting trauma to the triceps tendon occurred when the dog was attacked by a member of the public with a machete. Tendon transection was reported to have occurred $1 \mathrm{~cm}$ proximal to the olecranon tuber. Primary repair was attempted by the referring veterinarian 48 hours following this injury and included anastomosis of the triceps tendon using 0-PDS in a combination of simple continuous and interrupted cruciate sutures following wound flushing. Postoperative management included a modified Robert-Jones bandage and exercise

\section{(c) 2021. The Author(s).}

This is an open access article published by Thieme under the terms of the Creative Commons Attribution License, permitting unrestricted use, distribution, and reproduction so long as the original work is properly cited. (https://creativecommons.org/licenses/by/4.0/) Georg Thieme Verlag KG, Rüdigerstraße 14, 70469 Stuttgart, Germany 
restriction. Continued treatment with meloxicam (Boehringer Inglheim Pty Ltd, North Ryde, Australia) $(0.1 \mathrm{mg} / \mathrm{kg}$ per os [PO] every 24 hours) and strict confinement at home failed to improve a non-weight bearing left thoracic limb lameness.

The patient was then referred for further treatment 5 weeks after the initial trauma with no weight bearing reported since the inciting trauma. At that time the patient had been treated with 3 weeks of amoxycillin-clavulanic acid (Apex Laboratories Pty Ltd, Somersby, Australia) $(13 \mathrm{mg} / \mathrm{kg}$ PO every 12 hours) for a persistent draining tract proximal to the olecranon which was not resolving.

Upon presentation to the referral surgeon (R.G.C.), the patient was found to be non-weight bearing lame in the left forelimb with a purulent draining tract located $2 \mathrm{~cm}$ proximal to the olecranon on the caudolateral aspect of the distal brachium. Physical exam revealed a large palpable defect proximal to the olecranon. When the shoulder was fixed in extension, the elbow could be fully flexed, confirming complete incompetence of the triceps mechanism. Cutaneous innervation of the left forepaw remained intact circumferentially, indicating an intact nervous supply to the paw.

At this point, treatment was planned and divided into two stages.

1. Surgical exploration of the traumatized area with resection of sinus tract and interposed fibro-granulomatous tissue, lavage and exit culture in preparation for definitive reconstruction of the triceps brachii tendon.

2. Reconstruction and repair of the triceps brachii tendon once a healthy wound environment was achieved.

The previous surgical scar was excised and associated draining tract explored and debrided. The tract extended to the caudal distal humerus, just over the proximal olecranon. A large amount of proliferative granulation tissue and fibrous scar tissue proximal to the olecranon communicated with a draining tract to the skin. Aggressive en bloc resection of this reparative tissue and sinus was performed. Deep to this tissue was healthy fibrotic scar tissue interposed between the triceps brachii long head muscle belly and the olecranon. Following wound debridement and lavage, an exit culture was taken and submitted for routine culture and sensitivity. The subcutaneous layers and skin were closed routinely. The patient was discharged with instructions to administer meloxicam (Boehringer Inglheim Pty Ltd, North Ryde, Australia) ( $0.1 \mathrm{mg} / \mathrm{kg}$ PO every 24 hours), codeine (Aspen Pharma Pty Ltd, St. Leonards, Australia) $(0.8 \mathrm{mg} / \mathrm{kg}$ PO every 12 hours) and amoxycillin-clavulanic acid (Apex Laboratories Pty Ltd, Somersby, Australia) $(26 \mathrm{mg} / \mathrm{kg}$ PO every 12 hours) pending culture and sensitivity results. Strict exercise restriction was also instructed between surgical exploration and definitive repair.

The results of the final microbiology report showed occasional epithelial cells, with no bacteria seen on Gram/ZN stain and no growth obtained by day two of culture.

Definitive triceps brachii tendon repair was performed weeks after initial injury, 1-week post sinus and partial scar excision. A caudolateral approach to the left mid-distal brachium was performed. Fibrous adhesions between the lateral and long heads of the triceps brachii muscle were

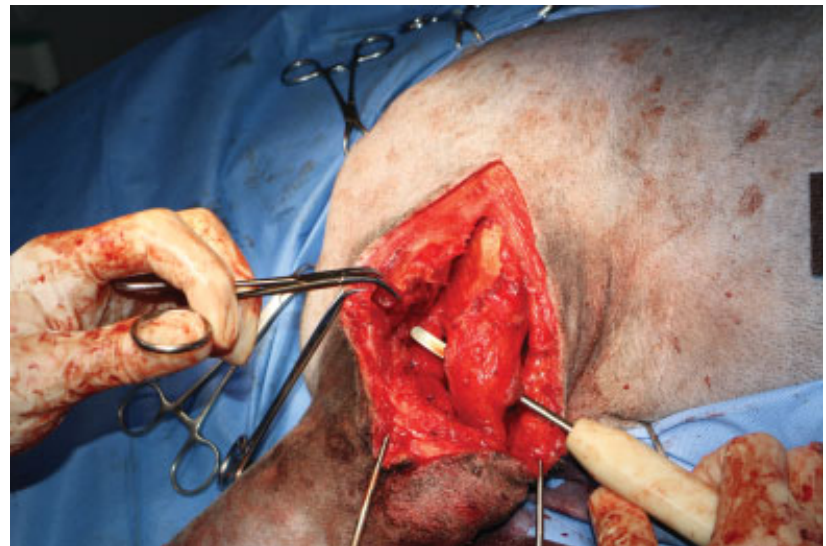

Fig. 1 Intraoperative photo of excessive scar tissue between the triceps brachii muscle and olecranon.

broken down. The accessory and medial heads were found encased in fibrous scar tissue along with the anconeus muscle. No attempt was made to dissect out these bellies. The radial nerve was identified and protected during dissection. The dysfunctional tendon remnants and the scar tissue (-Fig. 1) that was interposed between the muscle bellies of the lateral and long heads of the triceps were resected until healthy muscle was identified (-Fig. 2). Fibrous tissue present at the

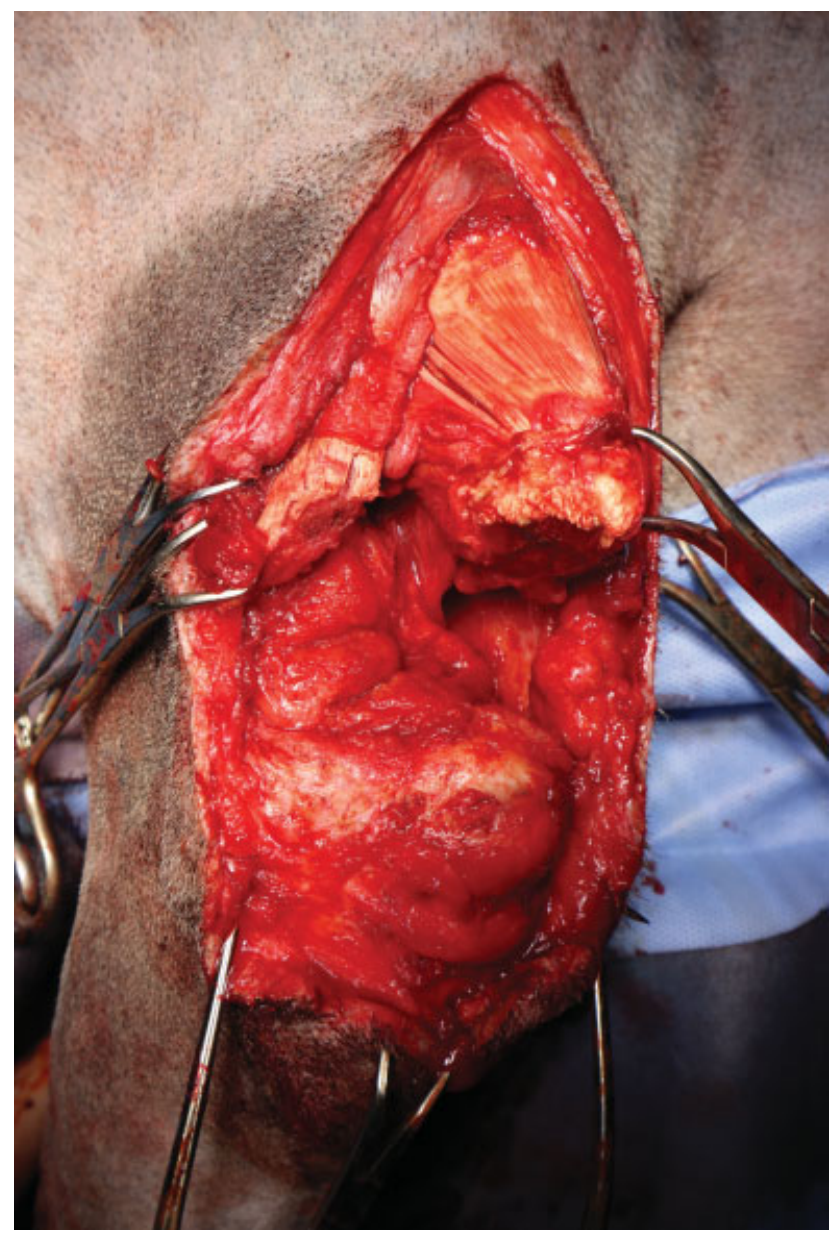

Fig. 2 Intraoperative photo following removal of all interposed fibroadipose scar tissue. 


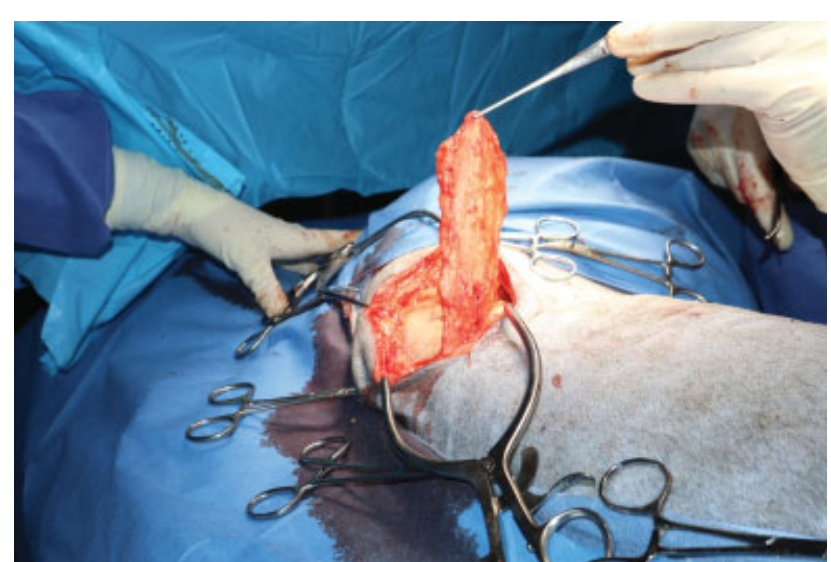

Fig. 3 Intraoperative photo of the harvesting of the thoracolumbar fascia (TLF) graft. The proposed margins were cranially at the level of the paralumbar fossa/caudal aspect of rib 13; caudally, just cranial to the tuber coxa in alignment with the aponeurosis of the sartorius muscle; dorsally, a parasagittal incision adjacent to the dorsal processes of the lumbar vertebrae and ventrally, dorsal to the aponeurosis of the TLF with the external and internal abdominal oblique muscles.

tendon insertion on the olecranon was also debrided, leaving $\sim 5 \mathrm{~mm}$ of tendon at the olecranon insertion point. With the elbow placed in full extension and the shoulder partly flexed, the long head muscle belly was attached to the stub of tendon on the olecranon with 0-Nylon using several locking loop sutures and three-loop pulley sutures. Due to marked tension of the anastomosis, the risk of postoperative suture pull-out and gap formation was considered very high. For this reason, a TLF graft was then harvested as previously described ( - Fig. 3 ) and placed over the repair as an onlay graft to support the anastomosis as a tension relieving graft. ${ }^{11}$ The TLF graft was oriented such that when harvested, its original cranial border was now positioned proximally over the triceps lateral head and its caudal border was now positioned over the triceps insertion point on the olecranon. The graft was secured with 0 PDS suture in a Krakow suture pattern running the length of the graft with its dorsal and ventral margins, now oriented cranially and caudally on the humerus respectively (-Fig. 4). The lateral head of the triceps was then secured to the

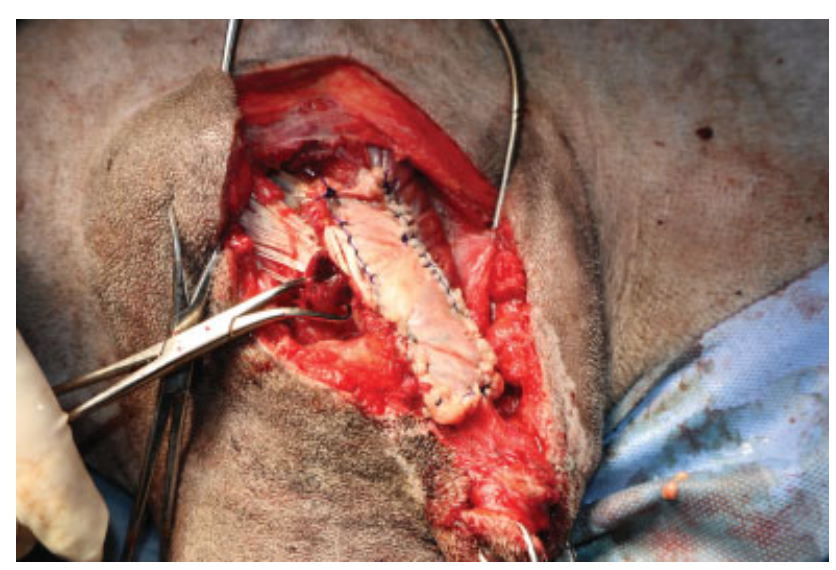

Fig. 4 The thoracolumbar fascia graft was secured with its dorsal and ventral margins, now oriented cranially (left) and caudally (right) on the humerus respectively. craniodistal border of the long head muscle and the onlay graft using 2-Nylon with a combination of locking loop and three loop pulley sutures. The site was lavaged thoroughly with sterile saline. The subcutaneous tissue was closed with 2-0 Glyconate with skin edges apposed with 3-0 Nylon in a Ford interlocking pattern.

The limb was bandaged, and a spica splint was fashioned from Dynacast Prelude Splinting Material; Roll $10 \times 4.6 \mathrm{~m}$ (BSN medical ANZ, Mulgrave, Australia) and applied postoperatively to protect and immobilize the left thoracic limb with the elbow maintained in an extended position for 6 weeks. A constant rate infusion (CRI) of fentanyl (Hameln Pharmaceuticals GmbH, Hameln Germany) (4 $\mu \mathrm{g} / \mathrm{kg} /$ hour $)$ was started intraoperatively and maintained at that rate throughout the procedure and overnight. Postoperatively, a CRI of medetomidine (Jurox Pty Ltd, Rutherford, Australia) ( $2 \mu \mathrm{g} / \mathrm{kg} /$ hour) was started and maintained at this rate until the patient was discharged from hospital 24 hours after surgery. The patient was discharged with instructions to administer meloxicam (Boehringer Inglheim Pty Ltd, North Ryde, Australia) (0.1 mg/kg PO every 24 hours) and codeine (Aspen Pharma Pty Ltd, St. Leonards, Australia) $(0.8 \mathrm{mg} / \mathrm{kg}$ PO every 12 hours) for 7 days, amoxycillin-clavulanic acid (Apex Laboratories Pty Ltd, Somersby, Australia) $(26 \mathrm{mg} / \mathrm{kg}$ PO every 12 hours) for 14 days following surgery, as well as strict confinement to a small area, exercise restriction to controlled leash walks for elimination purposes only, and bandage care. Four weeks' worth of acepromazine (Ceva Animal Health Pty Ltd, Glenorie, Australia) $(0.6 \mathrm{mg} / \mathrm{kg}$ PO every 12 hours) was also dispensed.

Follow-up examinations were performed every 2 weeks until spica splint removal at 6 weeks, 1 -week post spica splint removal at 7 weeks and then at 7 months after surgery. At 6 weeks following definitive surgical repair, all external coaptation was removed. The reconstructed triceps tendon was palpated as thick and robust with inability to flex the elbow while maintaining shoulder extension. Owners were instructed to maintain the patient on leash walking for a further 3 weeks, increasing frequency and duration of walks prior to resuming unrestricted off leash activity 9 weeks following definitive surgery. Re-evaluation at 4 and 7 months post definitive repair revealed the patient to be free of lameness with normal stance and elbow range of motion ( - Video 1 ).

\section{Video 1}

Owner's check in video 3 months post definitive repair of chronic left triceps tendon laceration. Online content including video sequences viewable at:https:// www.thieme-connect.com/products/ejournals/html/ 10.1055/s-0041-1725056.

\section{Discussion}

In order for a myotendinous apparatus to function effectively, achieving and maintaining appropriate tendon length and strength is of critical importance. ${ }^{12-14}$ In most cases, surgical 
treatment is performed shortly after tendon injury. Repair is considered delayed when the disrupted tendon has been neglected for at least 4 weeks. ${ }^{15}$ Untreated, the tendinous ends may atrophy and retract from their insertion, leaving inadequate tissue for anatomical reconstruction and a wide gap occupied by excessive fibro-adipose scar tissue with poor suture holding strength, which needs to be excised before repairing the tendon. ${ }^{15,16}$ In cases where a tendon gap persists or for failed tendon repairs, autogenous, xenogenic and synthetic grafts have been utilized to provide additional support. $^{17,18}$

In the case reported herein, failure of the primary repair at index surgery to resist tension resulted in a large gap formation with exuberant interposed 'bridging scar' tissue formation. Debridement of interposed scar tissue resulted in a $10 \mathrm{~cm}$ gap between the triceps long and lateral heads and the olecranon. Definitive surgery to re-attach these muscle bellies to the olecranon resulted in marked anastomosis tension which was protected with an autogenous TLF onlay graft to prevent repeat gap formation occurring.

Onlay augmentation (reinforcement) grafts in human rotator cuff repair models have been shown to increase stiffness and, as a result of load sharing, decrease tendon gapping. ${ }^{19,20}$ Studies have reported conflicting evidence regarding the effect on load to failure. ${ }^{19-23}$ Shea and colleagues report that application of an onlay augmentation was estimated to share $34.7 \%$ of the global load applied to the tendon repair during cyclic subfailure loading, transferring some of that load directly to the bone and bypassing the repair. ${ }^{20}$ Clinically, demonstration of superior stiffness is a more relevant evaluation of the day-today response of the repair construct to subdestructive loads that are frequently seen with the rehabilitation process. ${ }^{19}$ Strength, in terms of load to failure, represents the ability of the repair to resist a one off destructive force, which is likely less relevant if the repair is further protected by limb immobilization. Rather in humans, repetitive, low-level loading of the rotator cuff repair with routine muscle contraction, even in a postoperative sling, is thought to be the likely mechanism of gap formation. ${ }^{24}$ Use of a polyurethane urea onlay mesh graft has successfully been used to augment the surgical repair of a chronic tendon rupture in the canine. ${ }^{4}$ Application of the TLF graft in an onlay (reinforcement) fashion in the dog reported herein is thought to have helped prevent gap formation at the repair site and resist displacement at these subdestructive loads.

In this patient, the decision to reinforce the repair with an onlay free graft of autologous TLF graft was elected due to the increased graft size when compared with tensor fascia lata. ${ }^{11}$ Furthermore TLF grafts demonstrate similar tensile strength and stiffness in material properties to that of fascia lata. ${ }^{11,25}$ Additionally, the chronic nature of the discharging wound which communicated with the disrupted tendon was concerning for active infection. For this reason, staged treatment with debridement/lavage and exit culture prior to definitive repair was performed. Active bacterial infection is known to be detrimental to tendon healing. ${ }^{26-28}$ Despite the negative exit culture, an autogenous graft was still favoured over a synthetic graft for risk of ongoing infection.
Henderson and colleagues demonstrated that TLF, like fascia lata, acts in an anisotropic manner during tensile testing, with greater strength and stiffness in the dorsoventral direction and proximodistal direction respectively. ${ }^{11}$ Due to the inherent shape and useable surface area of TLF, the graft was placed over the repair with its original cranial-caudal orientation now oriented proximal to distally bridging the tendon repair. The authors believe that despite the orientation being perpendicular to the grafts strongest orientation, physiologic loads likely did not exceed its failure point as suggested by the successful outcome. Further biomechanical testing would be required to assess the TLF as an onlay graft in terms of its applied orientation and useable length. Furthermore, the discrepancy in useable length of the graft when oriented in the craniocaudal versus dorsoventral planes is significant, which influences how much tissue is covered and incorporated in the onlay repair which ultimately may influence construct strength.

In a triceps tenotomy model in the dog, only $56 \%$ of original strength was achieved at 6 weeks following traditional suture repair, justifying the need for postoperative immobilization to protect the repair from excessive, destructive, weight-bearing forces. $^{25}$ Early and colleagues reported that transarticular external skeletal fixation and spica splints to be the most commonly applied measure of elbow immobilization following triceps tendon repair in dogs and cats respectively. ${ }^{1}$ A spica splint was used successfully for support of the primary tendon reconstruction in this patient, immobilizing the limb in anatomical position to facilitate early weight bearing and protect the repair by avoiding excessive tension at the suture site. Furthermore, the surgeon did not want transarticular external skeletal fixation pin tracts in the region of the non-vascularized graft for potential increased risk of infection. Other options that have been reported in the veterinary literature are custom-made orthotic braces, long lateral splints and Robert-Jones bandages, with the former two achieving good functional oucome. . $^{2,10}$

Previous reports suggest that chronic disruption of triceps tendon would be associated with a guarded prognosis. ${ }^{29,30}$ However, the outcome reported with the technique described herein resulted in return to excellent function. Although definitive conclusions cannot be drawn from a single case report, the authors believe that consideration for tenorrhaphy augmentation with a free TLF onlay graft should be considered in particularly challenging cases.

\section{Authors' Contributions}

R.G.C. performed the surgery and revised the current manuscript. C.J.W. wrote the manuscript and submitted to the journal.

\section{Conflict of Interest}

None declared.

\section{References}

1 Earley NF, Ellse G, Wallace AM, et al. Complications and outcomes associated with 13 cases of triceps tendon disruption in dogs and cats (2003-2014). Vet Rec 2018;182(04):108 
2 Echigo R, Fujita A, Nishimura R, Mochizuki M. Triceps brachii tendon injury in four Pomeranians. J Vet Med Sci 2018;80(05): 772-777

3 Yoon H-Y, Jeong SW. Traumatic triceps tendon avulsion in a dog: magnetic resonance imaging and surgical management evaluation. J Vet Med Sci 2013;75(10):1375-1377

4 Ambrosius L, Arnoldy C, Waller KR III, Little JP, Bleedorn JA. Reconstruction of chronic triceps tendon avulsion using synthetic mesh graft in a dog. Vet Comp Orthop Traumatol 2015;28(03): 220-224

5 Clarke SP, Jermyn K, Carmichael S. Avulsion of the triceps tendon insertion in a cat. Vet Comp Orthop Traumatol 2007;20(03): 245-247

6 Liehmann L, Lorinson D. Traumatic triceps tendon avulsion in a cat. J Small Anim Pract 2006;47(02):94-97

7 Anson LW, Betts CW. Triceps tendon avulsion in a dog: surgical management and zeroradiographic evaluation. J Am Anim Hosp Assoc 1989;25:655-658

8 Davies JV, Clayton Jones DG. Triceps tendon rupture in the dog following corticosteroid injection. J Small Anim Pract 1982; 23:779-787

9 Gilmore DR. Triceps tendon avulsion in the dog and cat. J Am Anim Hosp Assoc 1984;20:239-242

10 García-Fernández P, Quero Martín P, Mayenco A, Gardoqui M, Calvo I. Surgical management and follow-up of triceps tendon avulsion after repeated local infiltration of steroids: two cases. Vet Comp Orthop Traumatol 2014;27(05):405-410

11 Henderson ER, Friend EJ, Toscano MJ, Parsons KJ, Tarlton JF. Biomechanical comparison of canine fascia lata and thoracolumbar fascia: an in vitro evaluation of replacement tissues for body wall reconstruction. Vet Surg 2015;44(01):126-134

12 Bojrab MJ, Monnet E. Mechanisms of Disease in Small Animal Surgery. 3rd edition. Jackson, WY: Teton NewMedia; 2010

13 Boyer MI, Gelberman RH, Burns ME, Dinopoulos H, Hofem R, Silva MJ. Intrasynovial flexor tendon repair. An experimental study comparing low and high levels of in vivo force during rehabilitation in canines. J Bone Joint Surg Am 2001;83(06): 891-899

14 Gelberman RH, Boyer MI, Brodt MD, Winters SC, Silva MJ. The effect of gap formation at the repair site on the strength and excursion of intrasynovial flexor tendons. An experimental study on the early stages of tendon-healing in dogs. J Bone Joint Surg Am 1999;81(07):975-982

15 Ibrahim SA. Surgical treatment of chronic Achilles tendon rupture. J Foot Ankle Surg 2009;48(03):340-346

16 Us AK, Bilgin SS, Aydin T, Mergen E. Repair of neglected Achilles tendon ruptures: procedures and functional results. Arch Orthop Trauma Surg 1997;116(6-7):408-411
17 Shani J, Shahar R. Repair of chronic complete traumatic rupture of the common calcaneal tendon in a dog, using a fascia lata graft. Vet Comp Orthop Traumatol 2000;13:104-108

18 Houlton JEF, Dyce J. The use of polypropylene mesh for revision of failed repair of superficial digital flexor tendon luxation in three dogs. Vet Comp Orthop Traumatol 1993;6:129

19 Schlegel TF, Hawkins RJ, Lewis CW, Motta T, Turner AS. The effects of augmentation with Swine small intestine submucosa on tendon healing under tension: histologic and mechanical evaluations in sheep. Am J Sports Med 2006;34(02):275-280

20 Shea KP, Obopilwe E, Sperling JW, Iannotti JP. A biomechanical analysis of gap formation and failure mechanics of a xenograftreinforced rotator cuff repair in a cadaveric model. J Shoulder Elbow Surg 2012;21(08):1072-1079

21 Nicholson GP, Breur GJ, Van Sickle D, Yao JQ Kim J, Blanchard CR. Evaluation of a cross-linked acellular porcine dermal patch for rotator cuff repair augmentation in an ovine model. J Shoulder Elbow Surg 2007;16(5, Suppl):S184-S190

22 MacGillivray JD, Fealy S, Terry MA, Koh JL, Nixon AJ, Warren RF. Biomechanical evaluation of a rotator cuff defect model augmented with a bioresorbable scaffold in goats. J Shoulder Elbow Surg 2006;15(05):639-644

23 van der Meijden OA, Wijdicks CA, Gaskill TR, Jansson KS, Millett PJ. Biomechanical analysis of two-tendon posterosuperior rotator cuff tear repairs: extended linked repairs and augmented repairs. Arthroscopy 2013;29(01):37-45

24 Burkhart SS, Diaz Pagàn JL, Wirth MA, Athanasiou KA. Cyclic loading of anchor-based rotator cuff repairs: confirmation of the tension overload phenomenon and comparison of suture anchor fixation with transosseous fixation. Arthroscopy 1997;13(06):720-724

25 Dueland R, Quenin J. Triceps tenotomy: biomechanical assessment of healing strength. J Am Anim Hosp Assoc 1980;16:507

26 Thomopoulos S, Parks WC, Rifkin DB, Derwin KA. Mechanisms of tendon injury and repair. J Orthop Res 2015;33(06):832-839

27 Lichtnekert J, Kawakami T, Parks WC, Duffield JS. Changes in macrophage phenotype as the immune response evolves. Curr Opin Pharmacol 2013;13(04):555-564

28 Sugg KB, Lubardic J, Gumucio JP, Mendias CL. Changes in macrophage phenotype and induction of epithelial-to-mesenchymal transition genes following acute Achilles tenotomy and repair. J Orthop Res 2014;32(07):944-951

29 Nielsen C, Pluhar GE. Outcome following surgical repair of Achilles tendon rupture and comparison between postoperative tibiotarsal immobilization methods in dogs: 28 cases (1997-2004). Vet Comp Orthop Traumatol 2006;19(04):246-249

30 Corr SA, Draffan D, Kulendra E, Carmichael S, Brodbelt D. Retrospective study of Achilles mechanism disruption in 45 dogs. Vet Rec 2010;167(11):407-411 